\title{
Measured long-ranged attractive interaction between charged polystyrene latex spheres at a water-air interface
}

\author{
Wei Chen, ${ }^{1}$ Susheng Tan, ${ }^{2}$ Zhoushen Huang, ${ }^{1}$ Tai-Kai Ng, ${ }^{1}$ Warren T. Ford, ${ }^{2}$ and Penger Tong ${ }^{1, *}$ \\ ${ }^{1}$ Department of Physics, Hong Kong University of Science and Technology, Clear Water Bay, Kowloon, Hong Kong \\ ${ }^{2}$ Department of Chemistry, Oklahoma State University, Stillwater, Oklahoma 74078, USA \\ (Received 2 January 2006; revised manuscript received 11 July 2006; published 15 August 2006)
}

\begin{abstract}
We report results of a systematic experimental study of interactions between charged polystyrene (PS) latex spheres at a water-air interface. Optical observations of stable bonded particle clusters and formation of circular chainlike structures at the interface demonstrate that the interaction potential is of dipole origin. Atomic force microscopy (AFM) is used to examine the distribution of charge groups on the colloidal surface. AFM phase images show patchy domains of size $\sim 100 \mathrm{~nm}$ on the particle surface, indicating that the surface charge distribution of the PS spheres is not uniform, as is commonly believed. Such patchy charges can introduce fluctuating in-plane dipoles, leading to an attraction at short interparticle separations. A theoretical analysis is given to explain the mechanism for attractions between like-charged particles at the interface.
\end{abstract}

DOI: 10.1103/PhysRevE.74.021406

\section{INTRODUCTION}

When charged colloidal spheres are dispersed into an aqueous solution, a fraction of the ionic functional groups on the surface dissociate. A net charge remains on the particle surface, and the counterions in the aqueous phase form spherical clouds surrounding the charged particles. The electrostatic interaction potential between two such identically charged spheres is purely repulsive and has the screenedCoulomb form [1]

$$
U(r)=\frac{q^{2} \exp \left[-(r-2 a) / \lambda_{D}\right]}{4 \pi \epsilon_{0} \epsilon\left(1+a / \lambda_{D}\right)^{2} r},
$$

where $r$ is the sphere's center-to-center separation, $q$ is the effective charge carried by each particle, $a$ is the particle radius, $\epsilon_{0} \epsilon$ is the dielectric constant of the fluid, and $\lambda_{D}$ is the Debye-Huckel screening length. This screened-Coulomb repulsion together with the short-ranged van der Waals attraction determines the stability of colloidal dispersions $[1,2]$.

While the above-mentioned theory, which was derived originally by Derjaguin, Landau, Verwey, and Overbeek (DLVO), has been used for many years to calculate the stability and phase behavior of a variety of macromolecular systems $[1,3,4]$, recent experiments indicate that under some special circumstances there are attractive interactions rather than the screened-Coulomb repulsion between like-charged species ranging from simple colloidal particles [5-9] to complex cytoskeletal filamentous actin [10] and DNA [11]. Because of its fundamental interest and important implications in colloid science and biology, the paradox of like-charge attractions has been under intensive theoretical scrutiny for many years $[3,4,12-18]$. Understanding of such attraction is extremely important as it determines the stability and structure formation of a variety of macromolecules in aqueous solutions, including proteins and DNA. Such an understanding is also required for computer modelling, process control,

\footnotetext{
*Email address: penger@ust.hk
}

and various engineering applications of colloids from soft materials to biotechnology. While a satisfying explanation for the origin of like-charge attractions still remains outstanding, a careful examination of the experimental conditions reveals that the attractions occur when the charged particles (or macromolecules) and the screening counterions are in confined geometries such that the usual spherical symmetry of the counterion distribution is broken. For example, attractions between like-charged particles were found only when they are dispersed near a solid wall [5-9].

Similar attractions were also found between like-charged particles dispersed at liquid interfaces [19-22]. Charged colloidal particles dispersed at an aqueous interface are usually stabilized by a repulsive Coulomb interaction. Because one of the phases forming the interface (air) is made of a nonpolar substance that cannot sustain charge, the counterions in the aqueous phase are distributed asymmetrically around the particle, producing an effective dipole moment for each particle. If the charge distribution on the particle surface is uniform, the resulting dipole moment points downward perpendicular to the interface, leading to a long-ranged dipolar repulsion between the interfacial particles $[23,24]$. If the interfacial particles are confined in a small area, the mutual repulsion between the particles can induce ordering [25] and crystallization $[26,27]$. However, mesostructure formation of interfacial particles was observed without area confinement [19-21], suggesting that the interfacial particles also experience attractive interactions. While theoretical arguments [28-32] were given to link the observed attractions to the capillary forces resulting from deformations of the interface due to wetting or surface roughness of the interfacial particles, a satisfying explanation for the origin of interfacial distortions still remains illusive $[21,33]$.

On the experimental side, we find that the charged particles that are used most widely in the experiments showing the like-charge attractions at the interface $[19,20]$ or near a solid wall [6-9] are polystyrene (PS) latex spheres with different charge-stabilizing chemical functionality on the surface. Interfacial particles have to be hydrophobic in order to stay at the water-air interface. On the other hand, charge- 
stabilizing (hydrophilic) chemical functionality has to be introduced on the particle surface so that the particles can be dispersed and remain stable at the interface. This requires a delicate balance and PS latex spheres satisfy this requirement. They are representative of charged particles commonly used in colloid science. A crucial assumption made for charged polymer latex spheres is that the surface charge distribution of the particles is uniform, but the actual distribution of the surface charge groups has not been examined systematically. While previous experiments [19-21] revealed interesting mesostructure formation of particles at the interface, well-controlled experiments providing detailed information about the interaction potential $U(r)$ between the interfacial particles are rather limited. Direct measurements of the colloidal interactions, therefore, become essential for the understanding of the mechanism for like-charge attractions at the interface. A challenge to the experiment is that the interactions and dynamics of the interfacial particles are extremely sensitive to impurities at the interface [34]. Accurate measurements of $U(r)$ require well-controlled procedures to clean the interface and colloidal samples thoroughly so that a well-dispersed monolayer of particles can be made repeatedly at the interface.

In this paper, we report results of a systematic experimental study of interactions between charged polystyrene latex spheres at a water-air interface. Optical observations of stable bonded particle clusters and formation of circular chainlike structures at the interface demonstrate that the interaction potential is of dipole origin. Atomic force microscopy (AFM) is used to examine the distribution of the charge groups on the colloidal surface. Patchy domains are found on the colloidal surface, indicating that the surface charge distribution is not uniform as is commonly believed. Such surface heterogeneity introduces fluctuating in-plane dipoles, leading to an attraction at short interparticle distances. Some of the results have been reported briefly in a Letter [35].

The remainder of the paper is organized as follows. We first describe the apparatus and the experimental method in Sec. II. Optical and atomic force microscopic measurements are presented in Sec. III. Further theoretical discussions are given Sec. IV. Finally, the work is summarized in Sec. V.

\section{EXPERIMENT}

\section{A. Apparatus and sample preparation}

The experimental setup is shown in Fig. 1. The water-air interface is prepared using a homemade Teflon trough of rectangular shape with width $10 \mathrm{~cm}$, length $28 \mathrm{~cm}$, and height $1.0 \mathrm{~cm}$. There are two Teflon barriers placed on the top of the trough in parallel with the short side of the rectangular trough and they can slide along the long side of the rectangular trough. The distance between the two barriers determines the effective length of the water-air interface accessible to the interfacial particles and it can be varied from $25 \mathrm{~cm}$ down to $1.5 \mathrm{~cm}$. An optical window that allows observation of the interfacial particles from below is installed at the center of the bottom surface of the trough. The window holder consists of a stainless steel insert which raises the optical window to within $2 \mathrm{~mm}$ of the water surface. The top

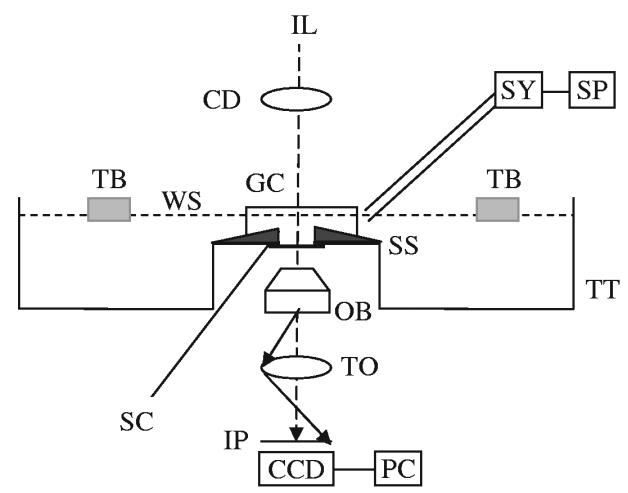

FIG. 1. Schematic diagram of the experimental setup (side view): IL, illuminating light; CD, condenser; GC, glass cover; WS, water surface; TB, two Teflon barriers; TT, Teflon trough; SS, stainless steel insert; SY, syringe; SP, syringe pump; SC, sample cell; $\mathrm{OB}$, objective; TO, tube optics; IP, image plane; CCD, chargedcouple device camera; $\mathrm{PC}$, host computer.

surface of the insert is cone shaped with a central hole of diameter $1.3 \mathrm{~cm}$. The bottom of the hole is sealed with a 0.1 -mm-thick glass cover slip, which serves as a window with thin optical path allowing the use of shorter focal length microscope objectives. The sidewall of the hole together with the bottom glass slip forms a sample cell that has an effective height of $1.0 \mathrm{~mm}$. The entire trough is placed on the sample stage of an inverted microscope (Leica DM-IRB), so that the motion of the interfacial particles can be viewed from below through an objective with magnification varied from $40 \times$ to $94.5 \times$. Higher magnifications are used for samples with higher particle concentrations.

Two kinds of polystyrene (PS) latex spheres are used in the experiment. One has anionic (negative) carboxyl groups on the surface with diameter $d=1.1 \pm 0.02 \mu \mathrm{m}$ and (nominal) surface charge density $\zeta_{0}=12.5 \mu \mathrm{C} / \mathrm{cm}^{2}$ (Interfacial Dynamics Corp., product number 7-1000). The second kind of PS spheres has anionic sulfate groups on the surface with diameter $d=1.0 \pm 0.03 \mu \mathrm{m}$ and $\zeta_{0}=2.8 \mu \mathrm{C} / \mathrm{cm}^{2}$ (Interfacial Dynamics Corp., product number 1-1000). The total charge of the sulfate-PS spheres is thus 5.4 times smaller than that of the carboxyl-PS spheres. The purchased aqueous samples are surfactant-free, but we find that the chemical impurities in the solution can still cause a substantial increase in the measured surface pressure isotherm. Surface pressure measurements of the supernatant solution (without particles) are carried out using a commercial Langmuir-Blodgett trough (Type-611, Nima).

To further clean the colloidal samples, we wash the particles with deionized water (or methanol) via repeated centrifugation. Two drops $(\sim 0.08 \mathrm{~mL})$ of the received aqueous solution with $4.1 \%$ solid concentration $(\mathrm{g} / \mathrm{mL})$ of particles are mixed with $5 \mathrm{~mL}$ deionized water $(18 \mathrm{M} \Omega)$. This solution is centrifuged at $2000 \mathrm{rpm}$ for $\sim 3 \mathrm{~h}$. After the centrifugation, the upper layer of supernatant is removed by a pipet and the remaining particles are mixed with another $5 \mathrm{~mL}$ of deionized water for the next centrifugation. The centrifugation speed and duration are chosen so that no particle aggregate is formed after the gentle centrifugation. The centrifu- 
gation process is repeated six to seven times until the final supernatant is tested to be clean. This is done by spreading the supernatant solution on a clean water-air interface and measuring its surface pressure isotherm using the commercial Langmuir-Blodgett trough. For cleaned supernatant solutions, their surface pressure rise is less than $0.1 \mathrm{mN} / \mathrm{m}$ when the interface area is reduced from 250 to $50 \mathrm{~cm}^{2}$. This is the sensitivity limit of the pressure sensor used in the experiment, indicating that no detectable impurity remains in the particle solution. The concentrated particle solution is further diluted with methanol at a desired concentration prior to use. An alternative way to clean the colloidal samples is to wash the particles directly with methanol. The centrifuge procedure remains the same as that with water except that the centrifuge time is reduced from $\sim 3 \mathrm{~h}$ to $\sim 20$ min each time. This is because the density difference between the PS spheres and methanol $\left(\Delta \rho \simeq 0.26 \mathrm{~g} / \mathrm{cm}^{3}\right)$ is 5.2 times larger than that with water $\left(\Delta \rho \simeq 0.05 \mathrm{~g} / \mathrm{cm}^{3}\right)$. As a result, the monodispersity of the centrifuged particle/methanol solution is found to be more sensitive to the centrifuge time.

A cleaning procedure [36] is followed to clean the homemade trough. First, the trough is cleaned with a Kimwipe (280 1-ply white wipers, Kimberly-Clark) soaked with acetone. The cleaned trough is then filled with deionized water. The water surface enclosed by the barriers is aspirated for approximately $1 \mathrm{~min}$ to further remove residual impurities on the surface. During the aspiration, the water surface is maintained above the sample cell. The cleaned particle/ methanol solution is then injected onto the water-air interface using a syringe pump (Model-100, KDS Scientific) with a 1-mL glass syringe (Hamilton) holding the particle/methanol solution. Typically, we keep the number concentration of the particle/methanol solution at $\sim 10^{8} / \mathrm{cm}^{3}$ and the injection rate is set at $15 \mu \mathrm{L} / \mathrm{min}$. Methanol drops wet the water surface quickly and the evaporation of the thin methanol layer only takes a few seconds. After the evaporation of methanol, a well dispersed monolayer of particles forms on the interface.

Initially, $\sim 300 \mu \mathrm{L}$ of the particle/methanol solution (the actual amount varies between 50 and $800 \mu \mathrm{L}$ ) are injected onto a large interfacial area $\left(\sim 8 \times 10 \mathrm{~cm}^{2}\right)$ to reduce the probability that the particles collide with each other during the volatile evaporation of methanol and form colloidal aggregates at the interface. Once dispersed onto the interface with a low surface coverage, the interfacial particles can be further concentrated by slowly pushing them into a smaller area with the two Teflon barriers on the trough. At a desired surface coverage, the water-air interface is lowered by pipetting water out from the bottom of the trough until the interface reaches the upper edge of the sample cell. In this way, a well-controlled monolayer of particles is maintained inside the sample cell. Because stainless steel is hydrophilic, the water surface attaches the sharp upper edge of the sample cell, which helps to pin down surface flows. The entire interface inside the sample cell is then covered by a glass cap to avoid the effect of air flows in the surroundings.

It is found that the experimental procedures described above are absolutely necessary to produce a well-behaved and nicely dispersed monolayer of particles at the interface. Without following these procedures, the interfacial particles

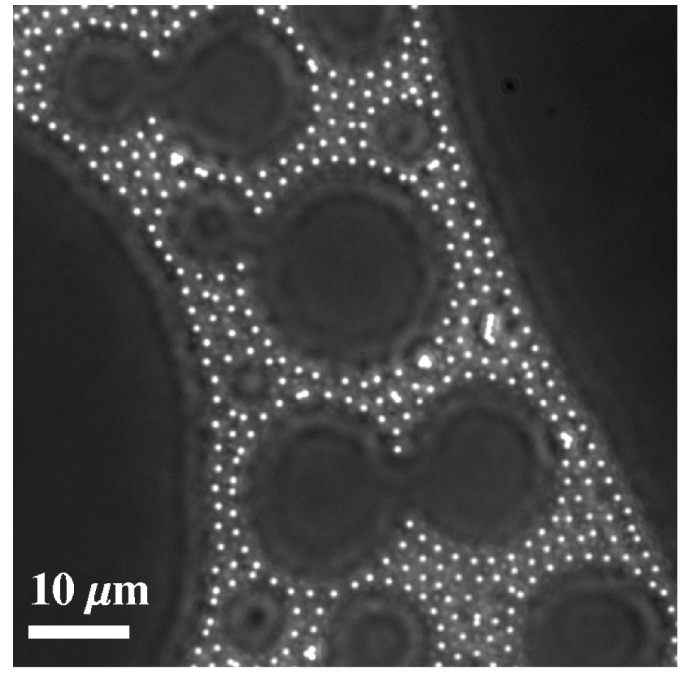

FIG. 2. Mesostructures of the carboxyl-PS spheres formed at the interface when the surface cleanness is not under a good control.

often form various mesostructures similar to those reported in Ref. [34] due to interfacial contamination by an invisible thin film of oil or some other impurities. An example is shown in Fig. 2. When contaminations at the interface are severe, the particles simply lie on the interface without any Brownian motion. For less severe interfacial contaminations, the motion of the interfacial particles is still hindered and their spatial configurations become "glassy" such that the measured pair correlation function $g(r)$ is not reproducible. By following the experimental procedures described above, we are able to remove impurities from the interface and produce a well-dispersed monolayer of particles at the interface. The individual particles undergo vigorous Brownian motion and remain stable at the interface for days. The mesostructures to be discussed below are introduced by well-controlled procedures and are completely reproducible. They are not produced by interfacial contaminations.

\section{B. Optical microscopy}

The motion of the interfacial particles is viewed with the Leica inverted microscope. Both the phase contrast and bright field microscopies are used to obtain good images of the particles undergoing vigorous Brownian motion. For high concentration samples, we find that the images obtained with the phase contrast appear to have slightly better contrast. For dilute samples, both the phase contrast and bright field work well. Usually, we use the phase contrast to obtain the particle images. Image sequences are recorded by a digital camera (CoolSNAP-cf, Thotometrics) and then stored into a host computer. The spatial resolution of each image is set at $1392 \times 1040$ pixels with a 12-bit dynamic range for the gray level. Typically, each particle occupies $\sim 50$ pixels in the image. The frame rate is set at one frame per second and the exposure time is $30 \mathrm{~ms}$.

Commercial image software (ImagePro, MediaCybernetics) is used for camera control and the determination of the particle positions in each image with a spatial resolution of 
60-100 nm. From the obtained particle positions, we calculate the pair correlation function $g(r)$ [37]

$$
g(r)=\frac{2 N(r)}{A n^{2} 2 \pi r d r-n \sum_{i}^{\text {edge }} \delta A_{i}(r)},
$$

where $N(r)$ is the number of particle pairs at separation $r$ in each image, $2 \pi r d r$ is the bin area, $n$ is the number density of the particles in the image, and $A$ is the area of the image. The last term in the denominator accounts for corrections due to the edge effect, where $\delta A_{i}(r)$ is the missing bin area of the $i$ th particle for large values of $r$ outside the image. Typically, we use 1000 images, each containing $\sim 100$ particles, to calculate $g(r)$. This corresponds to an average over 100000 particles, ensuring that the statistical average is adequate.

\section{Atomic force microscopy}

AFM measurements are performed on a layer of PS spheres, which is obtained by depositing a drop of a dilute aqueous suspension of particles on a newly cleaved mica substrate and drying at room temperature. The AFM topographic and phase images are obtained using a NanoScope IIIa Multimode scanning probe microscope (Digital Instruments, Veeco Metrology Group) operating in tapping mode under ambient conditions $\left(24 \pm 1{ }^{\circ} \mathrm{C}, 50 \pm 5 \%\right.$ relative humidity). Commercial silicon microcantilever probes (MikroMasch) with tip radius $5-10 \mathrm{~nm}$ and spring constant $2-5 \mathrm{~N} / \mathrm{m}$ are used in the AFM measurements. Topographic and phase images are obtained simultaneously at a driving frequency of $120 \mathrm{kHz}$ for the probe oscillation, and the scanning rate of the images is set at $1 \mathrm{~Hz}$. The AFM measurements are performed in the attractive regime with a fixed set-point ratio, $A_{\mathrm{sp}} / A_{0}=0.9$, where $A_{0}$ is the free oscillation amplitude (in air) and $A_{\mathrm{sp}}$ is a constant amplitude maintained during imaging.

\section{EXPERIMENTAL RESULTS}

\section{A. Optical microscopy measurements}

\section{Carboxyl polystyrene spheres}

Figure 3 shows two distinct configurations of the carboxyl-PS spheres observed at the interface. When the particle/methanol solution is injected onto the interface with low particle concentration and low injection rate (less than $50 \mu \mathrm{L} / \mathrm{min}$ ), a well dispersed layer of particles is obtained as shown in Fig. 3(a). The individual particles undergo vigorous Brownian motion and remain stable at the interface with various concentrations for days. Once dispersed onto the interface with a low surface coverage, these particles can be further concentrated by slowly pushing them into a smaller area with the two Teflon barriers on the trough. When the particle/methanol solution is injected onto the interface with higher particle concentration (larger than $10^{10} / \mathrm{cm}^{3}$ ) and/or higher injection rate (larger than $2 \mathrm{~mL} / \mathrm{min}$ ), the particles form bonded clusters as shown in Fig. 3(b), right after the evaporation of methanol. These particle clusters remain

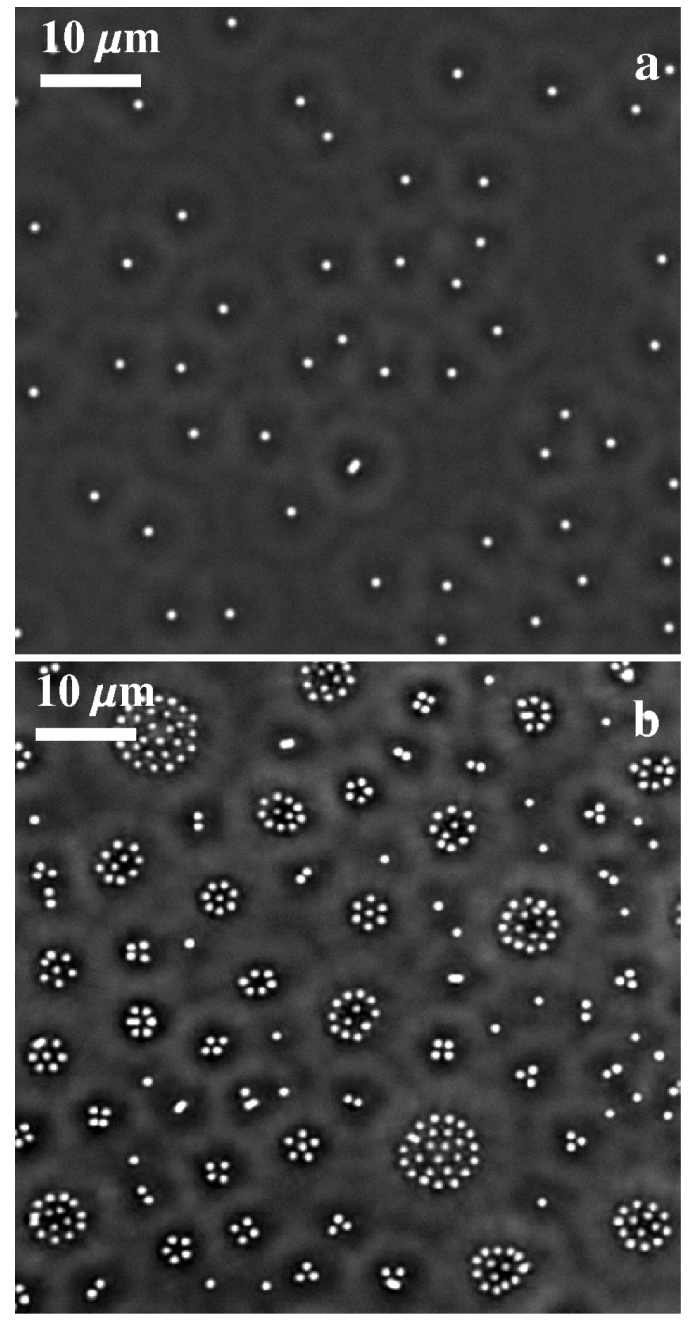

FIG. 3. (a) Equilibrium configuration of the carboxyl-PS spheres obtained with a low injection rate of the particle/methanol solution. (b) Particle configurations obtained with higher injection rates.

stable at the interface for days. By watching the motion of the particles in the large mesostructures over a period of time, we find that only the particles at the boundaries of the mesostructures form bonded chainlike structures and they arrange themselves like a pearl necklace along the contour of the mesostructures. Most particles in the interior of the mesostructures undergo Brownian motion freely but their overall motion is confined inside the mesostructures by the bonded particle chains at the boundary. We believe that the circular shape of the mesostructures results from the flow structures formed during the evaporation of methanol.

Using the image analysis software, we obtain the position of each particle shown in Fig. 3(a) and calculate their pair correlation function $g(r)$. Figure 4 shows the resulting $g(r)$ at three different values of the area fraction $\tilde{n}$ occupied by the particles. The measured $g(r)$ shows a strong dependence in $\widetilde{n}$, indicating that the repulsion between the carboxyl-PS spheres is very strong [38]. For dilute particle concentrations, the measured $g(r)$ is related to the interaction potential $U(r)$ through the Boltzmann equation 


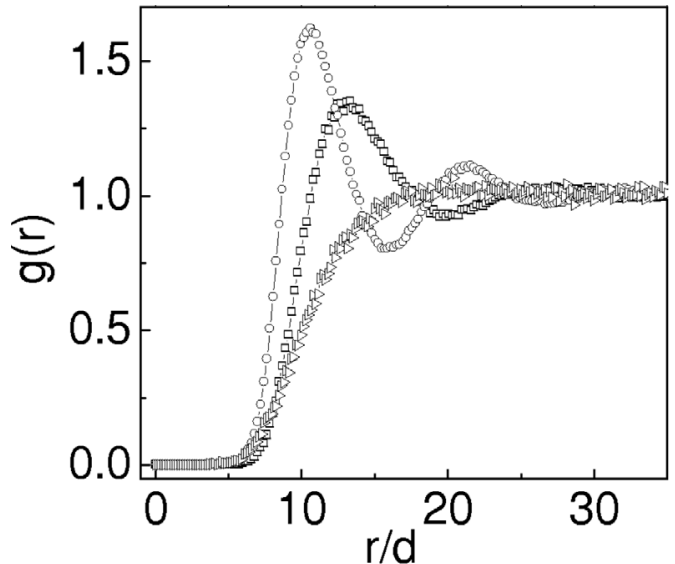

FIG. 4. Measured pair correlation function $g(r)$ of the carboxyl-PS spheres at three area fractions: $\tilde{n} \simeq 0.12 \%$ (triangles), $\tilde{n} \simeq 0.38 \%$ (squares), and $\tilde{n} \simeq 0.6 \%$ (circles).

$$
g(r) \simeq e^{-U(r) / k_{B} T}
$$

Figure 5 shows the interaction potential $U(r) / k_{B} T$ extracted directly from the measured $g(r)$ shown in Fig. 4. To avoid the crowding effect at finite particle concentrations, we calculate the many-body corrections to $U(r)$ using the hypernetted chain and Percus-Yevick approximations [37] and find that these corrections are negligible at the area fraction $\tilde{n}$ $\simeq 0.12 \%$. The circles in Fig. 5 show the calculated $U(r) / k_{B} T$ with the Percus-Yevick corrections. It is seen that they agree well with the triangles, which are obtained directly using Eq. (3). The solid curve in Fig. 5 shows the fitted function $U(r) / k_{B} T=B(d / r)^{3}$ with $B=1008$. Figure 5 thus demonstrates that the interaction potential between the charged interfacial particles at large separations is determined mainly by the dipole-dipole repulsion $[23,24]$. In a recent experi-

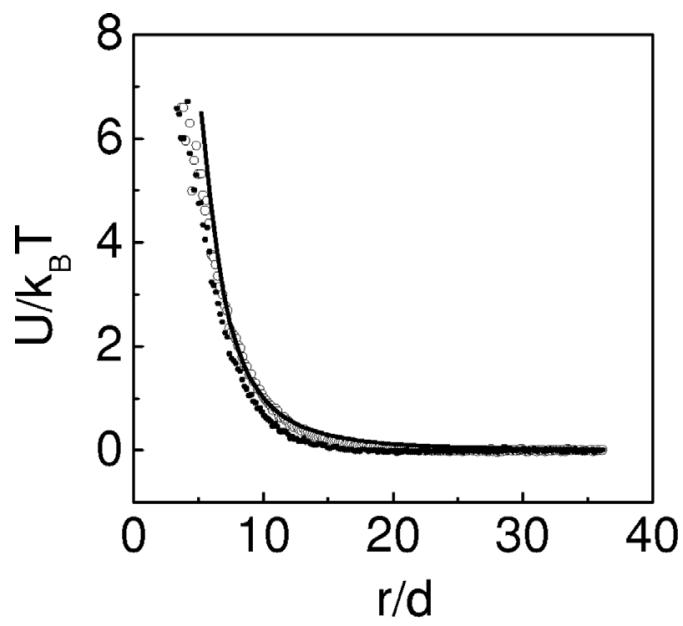

FIG. 5. Repulsive potential $U(r) / k_{B} T$ as a function of $r / d$ extracted from the measured $g(r)$ at $\tilde{n} \simeq 0.12 \%$ shown in Fig. 4 . The open circles are obtained with the Percus-Yevick corrections. The closed circles are obtained using the Boltzmann equation $U(r) / k_{B} T=-\ln [g(r)]$. The solid curve is a fit to the open circles with the fitting function $U(r) / k_{B} T=1008(d / r)^{3}$. ment [39], Aveyard et al. used laser tweezers to measure the long-ranged repulsive force between two charged PS spheres at an oil-water interface. Their results also indicated that the sphere's effective interaction potential $U(r)$ at large $r$ decays as $1 / r^{3}$.

In the experiment, we typically use $\sim 1000$ frames of particle images as a record to calculate $g(r)$. The resulting $g(r)$ is then averaged over many records (typically 10) taken at different locations of the sample. Among the particle samples prepared at different times, we find that the obtained $U(r)$ all decays as $1 / r^{3}$. The interaction amplitude, however, varies from sample to sample and the fitted value of $B$ is found to be in the range between 850 and 1200 . Some of the experimental uncertainties are caused by the number fluctuations in the two-dimensional colloidal sample. Considerable particle number fluctuations are found in the dilute samples from which we obtain $g(r)$. Another source of experimental uncertainties comes from the fact that the ionic strength of the colloidal sample is not actively controlled in the experiment. Slow dissolution of ions into the aqueous phase from atmospheric $\mathrm{CO}_{2}$ may change the value of the Debye screening length $\lambda_{D}$. In fact, we find that the colloidal samples made of fresh deionized water tend to have a larger value of $B$.

As mentioned above, the equilibrium particle configuration shown in Fig. 3(a) is stable even at higher concentrations when the particles are slowly pushed into a smaller area. This suggests that there may exist an energy barrier with a height of several $k_{B} T$, which prevents the particles from reaching a deep attractive well at a smaller interparticle separation. Such an attractive well is needed to hold the particles together, forming stable bonded clusters as shown in Fig. 3(b). For particles to reach the deep energy well, extra energy must be supplied to overcome the energy barrier. When the particle/methanol solution is injected onto the interface, the evaporation of methanol generates a strong nonuniform surface flow, which can produce large enough relative velocities for the particles at the interface to overcome the energy barrier and form bonded clusters through an uncontrolled nonequilibrium process. This mechanism can explain the mesostructure formation of interfacial particles as shown in Fig. 3(b) and those reported in early experiments $[19,20]$.

To test the mechanism, we carry out a controlled experiment to supply kinetic energy to the particles at the interface. We start with an equilibrium configuration as shown in Fig. 3(a) and generate a nonuniform surface flow by pipetting a small amount of water $(\sim 0.5 \mathrm{~mL})$ in and out of the aqueous phase, as indicated in Fig. 6(a). The periodic pipetting produces nonuniform flows both in the aqueous phase and at the interface, causing it buckling periodically. After the periodic pipetting, the particle configuration changes from Figs. 3(a) to 6(b). A typical value of the relative velocity between the particles at the interface can be estimated as $v \simeq f \sqrt{\Delta s}$, where $\Delta s \simeq 1.3 \mathrm{~cm}^{2}$ is the areal change of the interface during the buckling and $f \simeq 1 \mathrm{~Hz}$ is the frequency of the periodic pipetting. Such a relative velocity corresponds to a kinetic energy of $m v^{2} / 2 \simeq 9 k_{B} T$ for the PS spheres (with mass density $1.05 \mathrm{~g} / \mathrm{cm}^{3}$ and diameter $1.1 \mu \mathrm{m}$ ) to overcome the energy barrier. Figure 6 thus suggests that the energy barrier height 


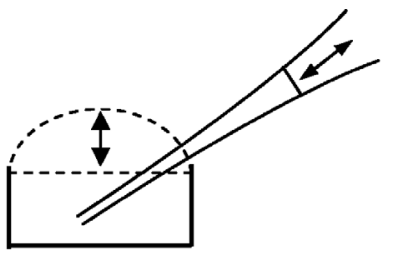

a

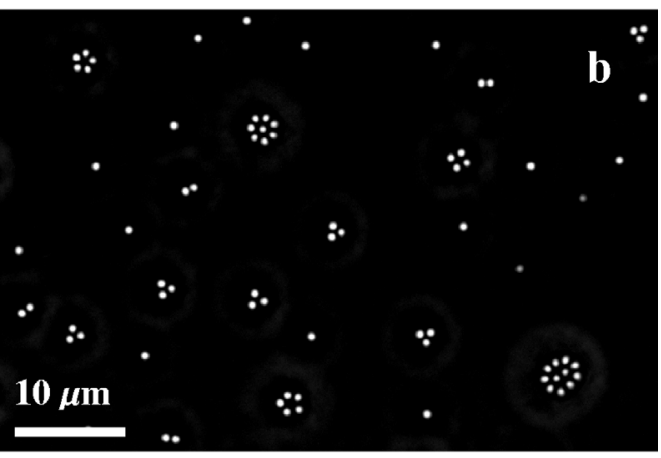

FIG. 6. (a) Operational schematic of the periodic pipetting. The dashed curves indicate the change of the interface when a small amount of water $(\sim 5 \mathrm{~mL})$ is pipetted in and out of the aqueous phase. (b) Formation of stable bonded particle clusters after the periodic pipetting.

for the carboxyl-PS spheres is of the order of $9 k_{B} T$.

Figure 7 shows the configurations of different particle clusters formed after the periodic pipetting. These particle clusters remain stable at the interface for days, indicating that the particles inside each cluster are trapped in a deep energy well. There is a significant separation between the neighboring particles within each cluster, making it different from particle aggregates in which all the particles are stuck together by van der Waals forces. The average separation $r_{0}$ between the particles varies slightly among the clusters with different particle numbers. For two-particle clusters, we find $r_{0} \simeq(2.0 \pm 0.3) d$. For five-particle clusters, we have $r_{0}$ $\simeq(2.2 \pm 0.5) d$. Such a large particle separation suggests that there exists another strong repulsion between the particles at distances smaller than $r_{0}$. This repulsion balances the longranged attraction, to be discussed below.

From the above measurements, we arrive at the following qualitative picture for the interaction potential $U(r)$. As de-
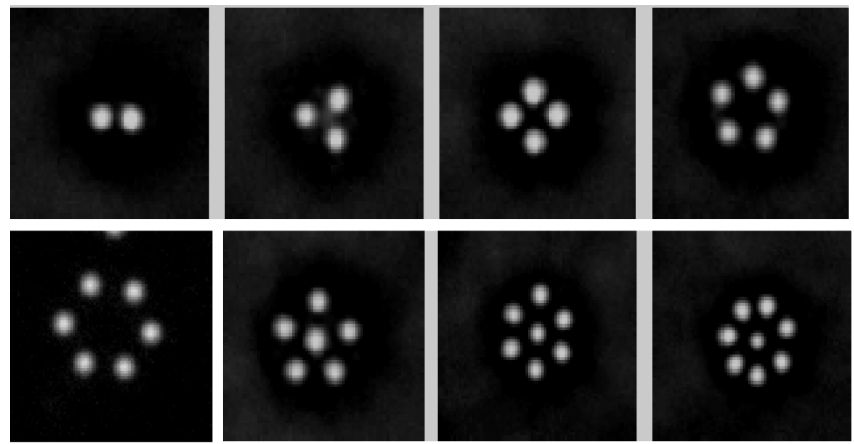

FIG. 7. Enlarged images of the individual bonded clusters of the carboxyl-PS spheres at the interface. These particle clusters are formed after the periodic pipetting. Particle size of $1.1 \mu \mathrm{m}$ serves as a scale bar.

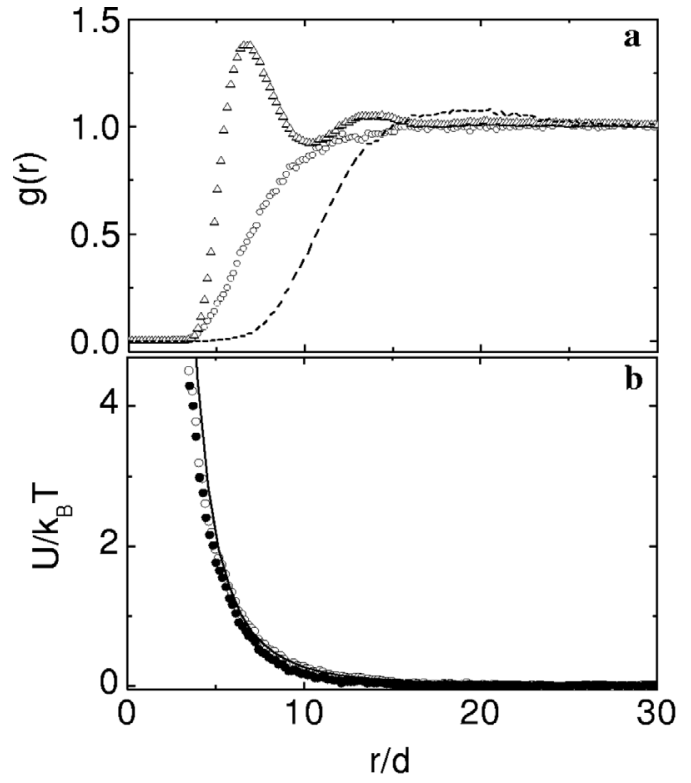

FIG. 8. (a) Measured pair correlation function $g(r)$ of the sulfate-PS spheres at two area fractions: $\widetilde{n} \simeq 0.16 \%$ (circles) and $\tilde{n}$ $\simeq 1.46 \%$ (triangles). For comparison, the measured $g(r)$ of the carboxyl-PS spheres at $\tilde{n} \simeq 0.16 \%$ is also shown (dashed curve). (b) Repulsive potential $U(r) / k_{B} T$ as a function of $r / d$ extracted from the measured $g(r)$ at $\widetilde{n} \simeq 0.16 \%$ shown in (a). The open circles are obtained with the Percus-Yevick corrections. The closed circles are obtained using the Boltzmann equation $U(r) / k_{B} T=-\ln [g(r)]$. The solid curve is a fit to the open circles with the fitting function $U(r) / k_{B} T=270(d / r)^{3}$.

picted in Fig. 13 below, it has an energy barrier of height $5-10 k_{B} T$ at $r_{1}$ and decays as $1 / r^{3}$ for larger $r\left(>r_{1}\right)$. For smaller particle separations, $U(r)$ has a deep energy well of the order of $10 k_{B} T$ at $r_{0}$. For even smaller separations $(r$ $<r_{0}$ ), the usual screened Coulomb repulsion is expected to dominate.

The interaction potential $U(r)$ only considers the twobody interactions. The observed "Coulombic molecules" shown in Fig. 7 exhibit various molecular symmetries, which result from a unique many-body effect for the attraction between the interfacial particles. For example, five particles arrange themselves in a symmetric pentagon shape. Six particles have two "degenerate" states. One is a ringlike structure with sixfold symmetry and the other has fivefold symmetry with an extra particle in the center of the cluster. It will be shown below that the various symmetric particle configurations shown in Fig. 7 result from minimizing the total electrostatic energy of the entire cluster. Some of the particle configurations shown in Fig. 7 were also observed in previous experiments $[19,21,35]$.

\section{Sulfate polystyrene spheres}

Similar to the carboxyl polystyrene spheres, the sulfate polystyrene spheres also show a dipole-dipole repulsion at large interparticle separations. Figure 8(a) shows the measured pair correlation function $g(r)$ of the sulfate-PS spheres at two area fractions. The measured $g(r)$ exhibits a weaker 

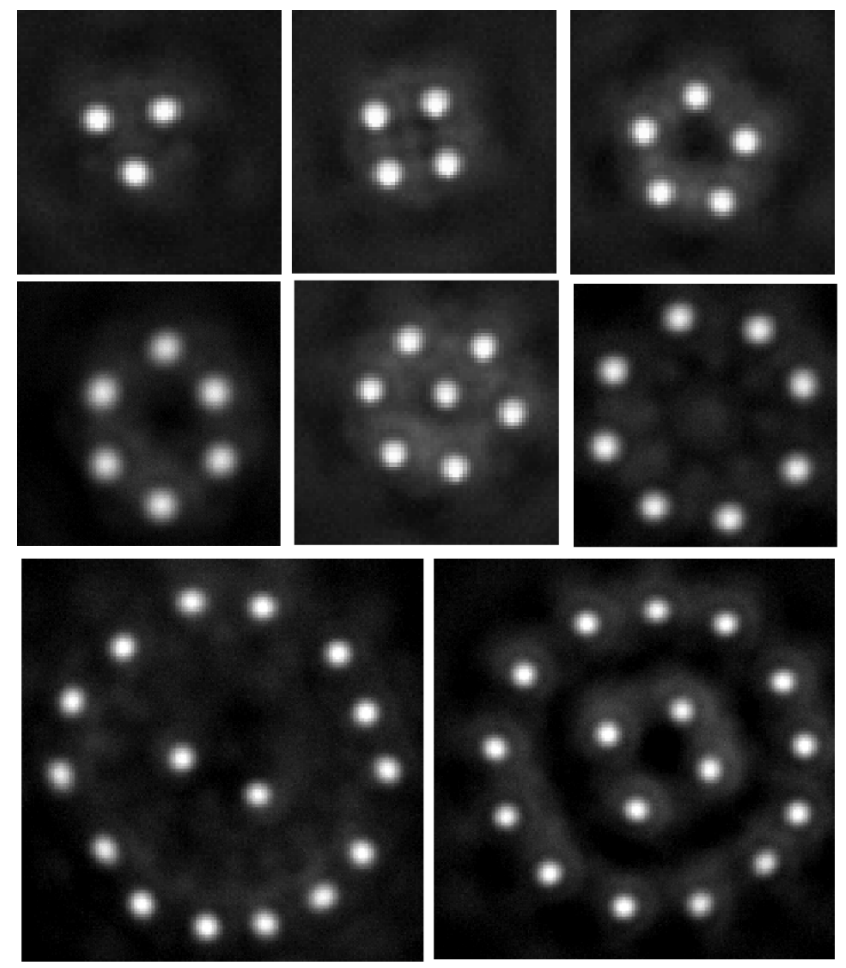

FIG. 9. Enlarged images of the individual bonded clusters of the sulfate-PS spheres at the interface. These particle clusters are formed after the periodic pipetting. Particle size of $1.0 \mu \mathrm{m}$ serves as a scale bar.

dependence on the area fraction $\widetilde{n}$, when compared with the carboxyl-PS spheres. If an effective hard-sphere diameter $\tilde{d}$ is defined as the interaction range in which $g(r) \simeq 0$, we find that the value of $\widetilde{d}$ for the sulfate-PS spheres is $\widetilde{d}_{\text {sul }} \simeq 3.8 d$ and that for the carboxyl-PS spheres is $\tilde{d}_{\text {car }} \simeq 7 d$. The shorter interaction range is caused by the fact that the sulfate-PS spheres carry fewer charges than the carboxyl-PS spheres do.

Figure 8(b) shows the interaction potential $U(r) / k_{B} T$ extracted from the measured $g(r)$ at $\widetilde{n} \simeq 0.16 \%$. The open circles show the calculated $U(r) / k_{B} T$ with the Percus-Yevick corrections. They agree well with the closed circles, which are obtained directly by using Eq. (3). The solid curve shows the fitted function $U(r) / k_{B} T=B(d / r)^{3}$ with $B=270$. This value of $B$ is approximately 3.7 times smaller than that obtained from the carboxyl-PS spheres. For the sulfate-PS spheres, we find that the sample-to-sample variations of the fitted value of $B$ are in the range between 200 and 350 . Figure 8(b) thus further confirms that the interaction potential between the charged PS spheres at the interface is determined mainly by the dipole-dipole repulsion when the interparticle separation is large.

The formation of bonded particle clusters is also observed in the sulfate-PS sphere system. Figure 9 shows the enlarged images of the individual bonded clusters formed after the periodic pipetting. The bonded particle clusters shown in Fig. 9 exhibit similar symmetries to those shown in Fig. 7. However, the average separation $r_{0}$ between the neighboring particles shown in Fig. 9 is larger than that shown in Fig. 7. For two-particle clusters, we find $r_{0} \simeq(2.5 \pm 0.5) d$ for the sulfate-PS spheres. For five-particle clusters, we have $r_{0}$ $\simeq(3.1 \pm 0.6) d$. Note that the variations in $r_{0}$ quoted here do not result from the statistical errors of the experiment. They are produced mainly by the Brownian motion of the particles within each cluster and thus can be treated as the rms value of $r_{0}$. The larger values of $r_{0}$ and its standard deviation suggest that the energy well for the sulfate-PS spheres is wider and shorted when compared with that for the carboxyl-PS spheres.

In the experiment, we observe that the carboxyl-PS and sulfate-PS spheres both form many ringlike structures either after the periodic pipetting or right after the evaporation of methanol (when a higher injection rate is used). An example is given in the sixth panel of Fig. 9. Often we find larger rings with a few particles moving freely at the center of the ring. By watching the motion of the particles, one can readily identify that these free particles do not belong to the particle cluster. This kind of ringlike structure is shown in the last two panels of Fig. 9. The formation of such stable circular chains is a hallmark of dipolelike attractions under no external field (more detailed discussions are given in Sec. IV C below).

\section{B. Atomic force microscopy measurements}

To examine the actual distribution of the surface charge groups, we conduct atomic force microscopy (AFM) measurements of surface topology of the individual PS spheres and phase shifts of the oscillating AFM cantilever. The AFM measurements are performed on a dry layer of PS spheres adsorbed on a clean mica substrate. Figure 10(a) shows the height image (two-dimensional) of a monolayer of the carboxyl-PS spheres. Figure 10(b) shows a magnified image (three-dimensional) of a sphere's top surface that is smoothed down to a few nanometers. The smooth top surface is seen more clearly in Fig. 10(d), which shows a crosssectional view of the height image of the sphere's top surface (curve 1). The sulfate-PS spheres also have a smooth surface similar to that of the carboxyl-PS spheres. Figure 11(a) shows a magnified height image (three-dimensional) of the smooth top surface of a sulfate-PS sphere. Figure 11(c) shows a cross-sectional view of the height image of the sphere's top surface (curve 1).

The phase images shown in Figs. 10(c) and 11(b) provide direct information about the domains of different chemical composition of the particle surface. It was shown [40,41] that the motion of the AFM cantilever in the tapping mode can be approximately described by the equation of motion for a driven harmonic oscillator,

$$
m \frac{\partial^{2} z}{\partial t^{2}}+c \frac{\partial z}{\partial t}+k z=F_{0} \cos (\omega t)+F_{\mathrm{ts}}(z)
$$

where $z$ is the tip deflection, $m$ and $k$ are, respectively, the effective mass and the spring constant of the cantilever, and $c$ represents the viscous damping. The external forces consist of the tip-sample interacting force $F_{\mathrm{ts}}(z)$ and the driving force $F_{0} \cos (\omega t)$, where $F_{0}$ is the amplitude and $\omega$ is the 

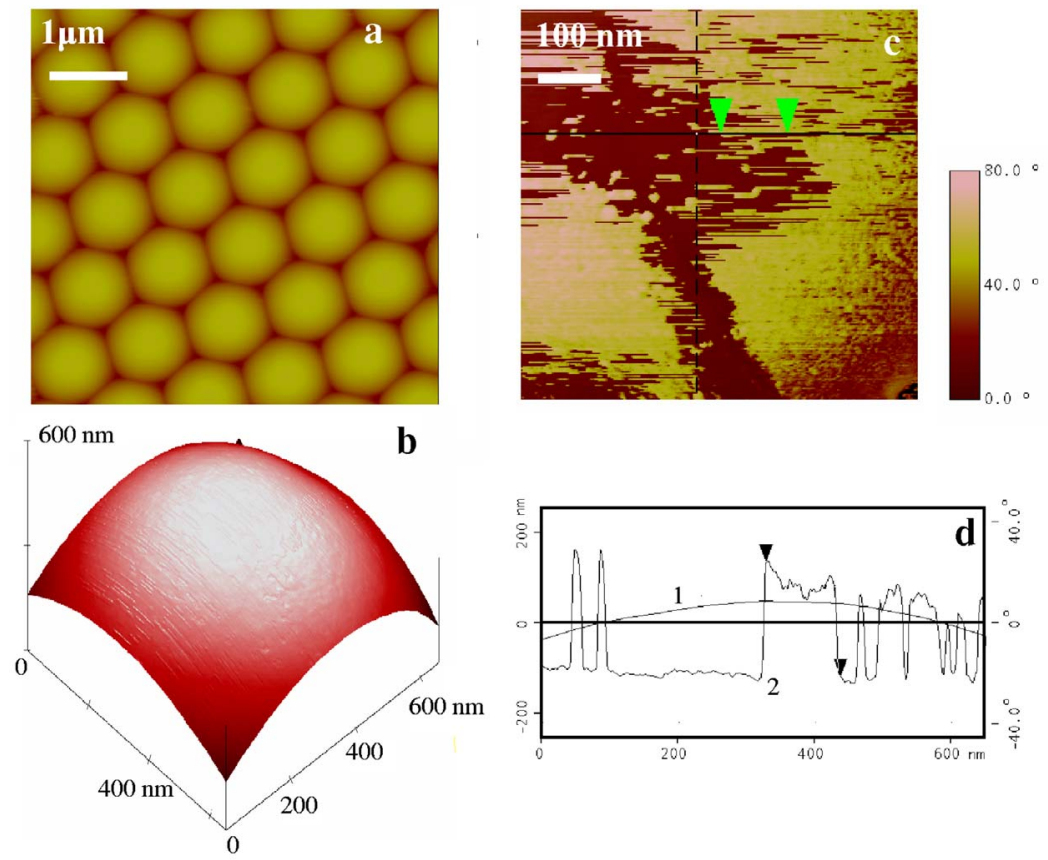

FIG. 10. (Color online) AFM measurements on the carboxyl polystyrene spheres. (a,b) AFM topographic images with two different scan sizes. (c) AFM phase image of the sphere's top surface simultaneously recorded with (b). (d) Crosssectional view of the measured height image (curve 1) and phase image (curve 2) of the sphere's top surface. forced oscillation frequency. The steady-state solution of Eq. (4) is given by [40]

$$
z\left(z_{c}, t\right)=z_{0}\left(z_{c}\right)+A\left(z_{c}\right) \cos \left[\omega t-\varphi\left(z_{c}\right)\right],
$$

where $z_{0}, A$, and $\varphi$ are, respectively, the mean deflection, amplitude, and phase shift of the cantilever oscillation. The parameter $z_{c}$ describes the equilibrium tip-sample separation in the absence of $F_{\mathrm{ts}}$. With the cantilever tip in the attractive regime $z_{c} \simeq 2-3 \mathrm{~nm}$ above a sphere's top surface, the phase delay $\varphi$ of the oscillating cantilever is determined primarily by the van der Waals attraction between the cantilever tip and the almost flat top surface [40,41]. Because hydrophilic carboxyl-PS domains have a Hamaker constant different from that of hydrophobic neutral PS domains, the phase contrast images reveal the surface distribution of the ionizable carboxyl-PS groups [42].

Figure 10(c) shows the phase image simultaneously recorded with the height image shown in Fig. 10(b). We assign the yellow-green (lighter) regions with greater phase shift $(\varphi \simeq 2 \pi / 7)$ to the carboxyl-PS domains. Patchy regions of carboxyl-PS domains of size $\sim 100 \mathrm{~nm}$ are found on the particle surface, indicating that the surface charge distribution of the carboxyl-PS spheres is not uniform as is commonly believed. We have carried out extensive AFM measurements on five different PS samples with particle radius varied from 1 to $0.5 \mu \mathrm{m}$, charge density varied from 2.6 to $12.5 \mu \mathrm{C} / \mathrm{cm}^{2}$, and surface charge group varied from carboxyl to sulfate. Figure 11(b) shows a phase image obtained on the top surface of a sulfate-PS sphere. This phase image is taken simultaneously with the height image shown in Fig. 11(a). The surface coverage of the patchy regions is reduced because the sulfate-PS spheres carry fewer sulfate-PS groups (and hence fewer charges). The AFM measurements on the commercial samples together with those on our own synthesized PS samples [42] of quaternary ammo- nium, sulfonate, and hydroxyethyl methacrylate surface chemistry all reveal surface inhomogeneities similar to those shown in Figs. 10(c) and 11(b), suggesting that the observed surface heterogeneity is not simply a defect of a particular particle sample. Rather, it is a general behavior of the charged PS spheres, which we attribute to the surface phase separation between hydrophilic and hydrophobic chemical components.

\section{THEORETICAL DISCUSSIONS}

\section{A. Attraction between two fluctuating in-plane dipoles}

The formation of chainlike structures as shown in Figs. 7 and 9 together with the above AFM measurements clearly demonstrate that the attraction between the charged PS spheres is anisotropic in the plane of the interface. This finding prompts us to consider anisotropic attractions, such as electric dipole interactions, rather than the usual isotropic interactions for the interfacial particles. As mentioned above, charged colloidal particles dispersed at an aqueous interface are stabilized by the Coulomb repulsion between the induced out-of-plane dipoles. Figure 12 shows the surface charge on the particle and the asymmetric counterion clouds in water at an average separation of the Debye screening length $\lambda_{D}$ from the sphere's surface. The charges on the PS spheres' surface result from the dissociation of the counterions in the aqueous phase. Because the distribution of the counterion clouds is asymmetric with respect to the reflection about the plane of the interface, a dipole is generated that points downward perpendicular to the interface. Hurd [24] showed that the induced dipole moment has a magnitude $P_{z} \simeq q_{0} \lambda_{D} / \sqrt{\epsilon}$, where $q_{0}$ is the effective charge carried by the particle and $\epsilon$ $(\simeq 80)$ is the dielectric constant of water.

It should be noted that Hurd's calculation is based on a simple point dipole model without considering the size effect 

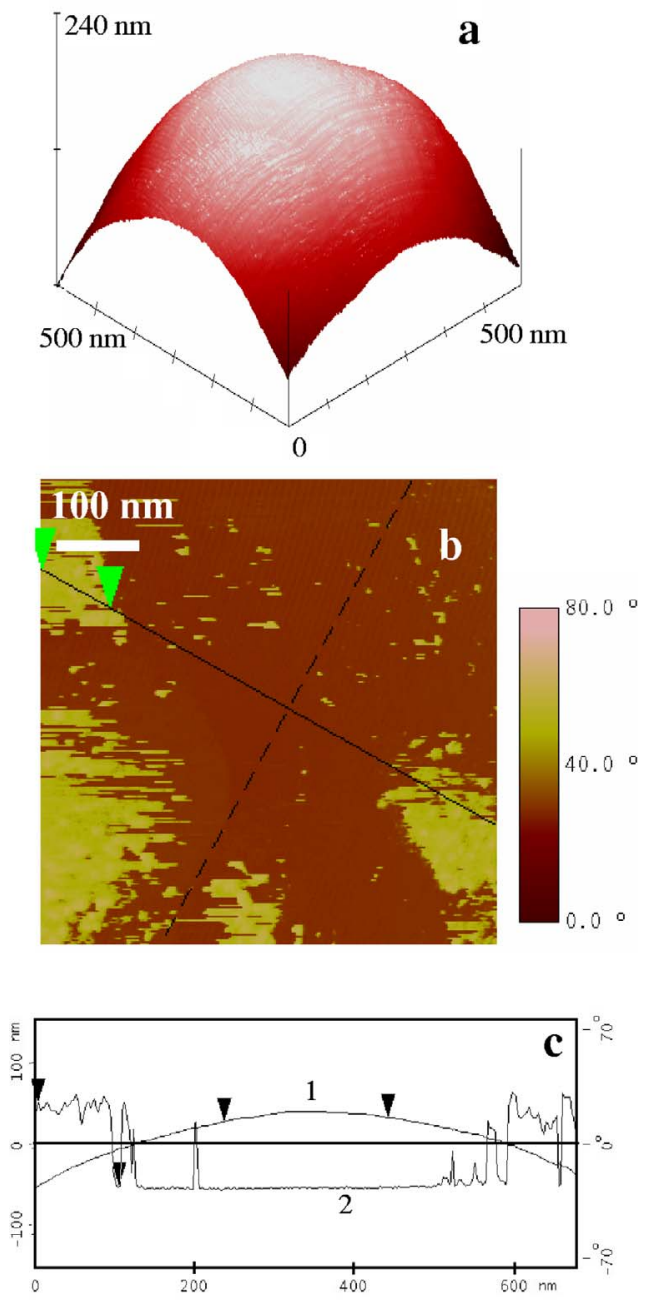

FIG. 11. (Color online) AFM measurements on the sulfate polystyrene spheres. (a) AFM topographic image of a sulfate-PS sphere (three-dimensional). (b) AFM phase image of the sphere's top surface simultaneously recorded with (a). (c) Cross-sectional view of the measured height image (curve 1) and phase image (curve 2) of the sphere's top surface.

of the particle, whose dielectric constant is different from those of air and water. In addition, the linearized PoissonBoltzmann equation was utilized for the electrostatic potential, which did not take the nonlinear effect of the PoissonBoltzmann equation into account. For highly charged colloidal particles, such as the PS spheres used in this experiment, the strong electrostatic coupling between the particle and the surrounding counterions results in a dense layer of counterions in the immediate vicinity of the particle, and thereby the particle behaves apparently as a new entity with an effective charge $q$ in Eq. (1) much smaller than the particle's bare charge $q_{\text {bare }}$ [43-45]. Aubouy et al. [46] derived an analytic expression showing the relationship between $q$ and $q_{\text {bare }}$ for the screened-Coulomb (monopole) interaction between two charged spheres fully immersed in the aqueous phase. In fact, from the fitting results shown in Figs. 5 and 8(b), one can also compute the effective charge $q_{0}$ from the obtained dipole moment $P_{z} \simeq q_{0} \lambda_{D} / \sqrt{\epsilon}$. For carboxyl-PS spheres, we find that the calculated surface charge density $\zeta_{0}$ using the fitted value of $q_{0}$ is 7.5 times smaller than the quoted value of $\zeta_{0}$ by the manufacturer. For less-charged sulfate-PS spheres, the calculated $\zeta_{0}$ is 3.1 times smaller than the quoted value of $\zeta_{0}$. This discrepancy is perhaps not surprising given that the charged spheres are at the interface and are not fully immersed in water. Clearly, a further theoretical analysis is needed to connect the measured effective dipole moment with the bare charge of the interfacial particles.

A crucial assumption made for all the charged latex spheres is that their surface charge distribution is uniform. If the charge distribution on the particle surface is not uniform, the patchy domains of surface charges will make additional contributions to the dipole moment. When the size of the patchy domains is too large to be averaged out spatially, such as those shown in Figs. 10(c) and 11(b), a net dipole moment is produced with a nonzero mean. The charged sphere at the interface rotates to maximize the number of patchy charges in contact with water (or equivalently, to minimize the total electrostatic energy) until the net charge-inhomogeneityinduced dipole points downward perpendicular to the interface. In addition, the patchy charges also produce fluctuating dipoles (with zero mean), which become particularly important in the plane of the interface, in which there exists no permanent dipole. As a result, the charged interfacial particles can be modeled to have a permanent out-of-plane dipole moment (solid arrow) and a fluctuating (in direction) in-plane dipole moment with a zero mean (dashed arrow), as indicated in Fig. 12. The in-plane dipole moment has a rms value $P_{\|} \simeq \alpha P_{z}$, where $0 \leqslant \alpha \lesssim 1$ is a numerical factor that depends on the surface heterogeneity.

With contributions from both the permanent out-of-plane dipole and the fluctuating in-plane dipole, the interaction potential between two (identical) interfacial particles can be written as [2]

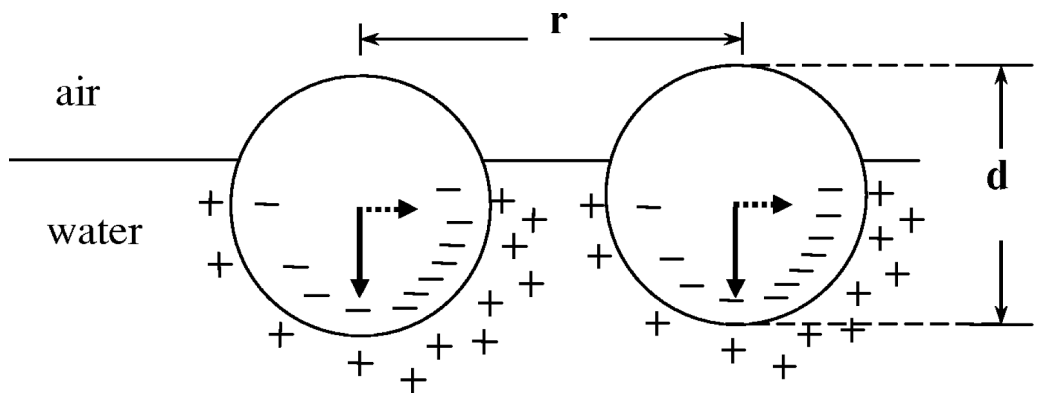

FIG. 12. Schematic of charged particles at the water-air interface and distributions of surface charge and counterions near the interface. The solid arrow shows a permanent out-of-plane dipole moment and the dashed arrow indicates a fluctuating in-plane dipole moment (with zero mean). 


$$
U(r)=\frac{A e^{-r / \lambda_{D}}}{4 \pi \epsilon_{0} \bar{\epsilon} r}-\frac{P_{\| f}^{2} f\left(\phi_{01}, \phi_{02}\right)}{4 \pi \epsilon_{0} \bar{\epsilon} r^{3}}+\frac{P_{z}^{2}}{4 \pi \epsilon_{0} \bar{\epsilon} r^{3}},
$$

where $\bar{\epsilon}[\simeq(\epsilon+1) / 2 \simeq \epsilon / 2]$ is the effective dielectric contact at the interface. The function $f\left(\phi_{01}, \phi_{02}\right)$ $=\left\langle 3 \cos \phi_{01} \cos \phi_{02}-\cos \left(\phi_{02}-\phi_{01}\right)\right\rangle$ describes the alignment between two fluctuating in-plane dipoles, whose orientations are specified by the angles $\phi_{01}$ and $\phi_{02}$ with respect to the vector connecting the two particles. On the right-hand side of Eq. (6), the first term represents the usual screened Coulomb repulsion between two charged spheres, as shown in Eq. (1) with $A=q^{2} \exp \left(2 a / \lambda_{D}\right) /\left(1+a / \lambda_{D}\right)^{2}$. The second term is the attraction between two fluctuating in-plane dipoles, and the third term is the repulsion between two permanent out-ofplane dipoles. van der Waals attraction is negligibly weak at the particle separations of interest and is omitted from Eq. (6).

For a given temperature $T$, one can define a characteristic separation

$$
\lambda_{N}=\left(\frac{2 P_{\|}^{2}}{4 \pi \epsilon_{0} \bar{\epsilon} k_{B} T}\right)^{1 / 3},
$$

at which the attractive dipole interaction becomes equal to the thermal energy $k_{B} T$. For $r>\lambda_{N}, k_{B} T$ is larger than the attractive dipole interaction and we have $P_{\|}^{2} f\left(\phi_{01}, \phi_{02}\right) /\left(4 \pi \epsilon_{0} \bar{\epsilon} r^{3}\right) \rightarrow 1 / r^{6}$. Because $1 / r^{6}$ decays faster than $1 / r^{3}$, the interaction potential is thus dominated by the dipole-dipole repulsion $\left[U(r) \sim P_{z}^{2} / r^{3}\right]$. For $d+\lambda_{D}<r \leq \lambda_{N}$, the fluctuating in-plane dipoles are aligned in line by the attractive interaction between each other, which is larger than $k_{B} T$, and thus $f\left(\phi_{01}, \phi_{02}\right) \simeq 2$. In this case, $U(r)$ becomes attractive if $P_{\|} \geqslant P_{z} / \sqrt{2}\left[U(r) \sim-\left(2 P_{\|}^{2}-P_{z}^{2}\right) / r^{3}\right]$. At even smaller separations with $r \sim d+\lambda_{D}, U(r)$ will be dominated by the usual screened Coulomb repulsion $[U(r) \sim \exp$ $\left.\left(-r / \lambda_{D}\right) / r\right]$. The balance between the first two terms on the right-hand side of Eq. (6) gives rise to the attractive well at $r_{0}$ (see Fig. 13), and that between the last two terms on the right-hand side of Eq. (6) produces the repulsive barrier at $r_{1}$.

\section{B. Numerical calculation of $U(r)$ for two identical spheres of finite size}

While Eq. (6) is correct only in the point dipole limit, the characteristic $r$ dependence of $U(r)$ discussed above is quite general. To take the particle size effect into account, we evaluate the electrostatic potential between two identical spheres numerically with a hypothetical nonuniform surface charge density. The calculation of $U(r)$ is carried out by assuming that there is a layer of charge with surface charge density $\zeta(\theta, \phi)$ on the surface of a sphere immersed in water and another layer of charge $-\zeta(\theta, \phi)$ at distance $\lambda_{D}$ from the sphere's surface in water. For simplicity, we further assume that the sphere is half immersed in water and the surface charge density takes a simple form

$$
\zeta(\theta, \phi)=\zeta_{0}\left[1+b \cos \left(\phi-\phi_{0}\right)\right]
$$

where $\zeta_{0}$ is the average surface charge density of the particle and $b$ is an adjustable parameter characterizing the surface

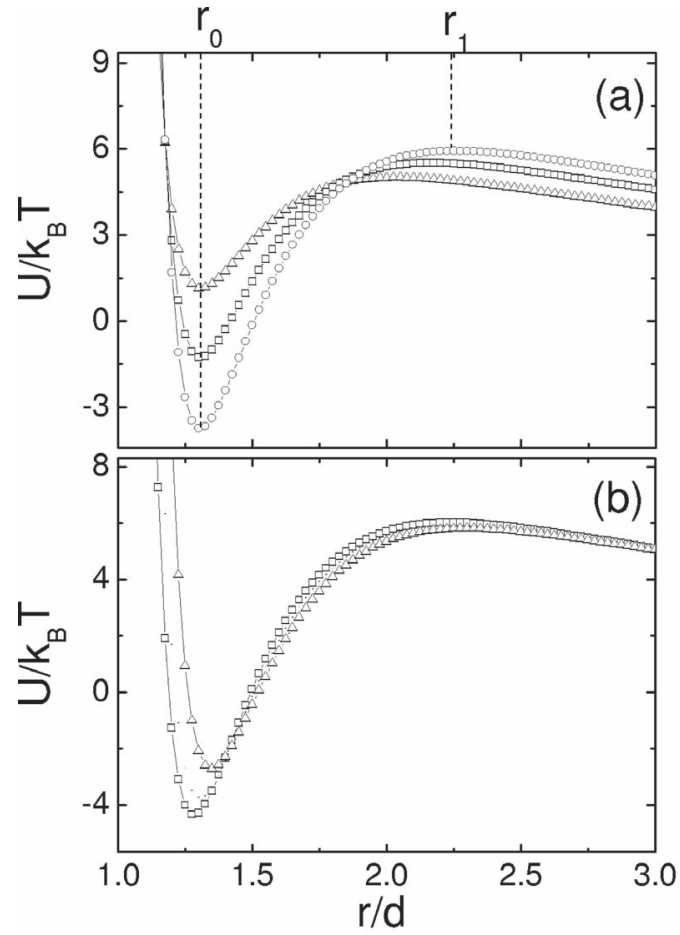

FIG. 13. Calculated interaction potential $U(r) / k_{B} T$ as a function of the normalized particle separation $r / d$. (a) Results obtained with fixed $b=0.88$ and $\lambda_{D}=20 \mathrm{~nm}$ but varying surface charge density $\zeta_{0}=1.1 \mu \mathrm{C} / \mathrm{cm}^{2} \quad$ (circles), $0.95 \mu \mathrm{C} / \mathrm{cm}^{2} \quad$ (squares), and $0.78 \mu \mathrm{C} / \mathrm{cm}^{2}$ (triangles). (b) Results obtained with fixed $b=0.88$ but varying Debye-Huckel screening length and surface charge density $\lambda_{D}=2 \mathrm{~nm}, \zeta_{0}=11 \mu \mathrm{C} / \mathrm{cm}^{2}$ (squares) and $\lambda_{D}=50 \mathrm{~nm}, \zeta_{0}$ $=0.44 \mu \mathrm{C} / \mathrm{cm}^{2}$ (triangles).

heterogeneity. In Eq. (8), $\theta$ and $\phi$ are the angular variables in the spherical coordinates with $\pi / 2 \leqslant \theta \leqslant \pi$ and $0 \leqslant \phi \leqslant 2 \pi$. Given the hypothetical surface charge density $\zeta(\theta, \phi)$, one can calculate the out-of-plane dipole moment $P_{z}$ $\simeq \pi a^{2} \zeta_{0} \lambda_{D} / \sqrt{\epsilon}$ and the in-plane dipole moment $P_{\|}$ $\simeq(\pi / 4) b \pi a^{2} \zeta_{0} \lambda_{D}$, pointing to the direction $\phi_{0}$ [47]. The value of $P_{z}$ is reduced by a factor of $\sqrt{\epsilon}$, because of the opposing image dipole across the interface. The ratio of the two dipole moments is thus $P_{\|} / P_{z}=\pi b \sqrt{\epsilon} / 4$.

The electrostatic potential $V\left(r, \phi_{01}, \phi_{02}\right)$ between sphere 1 and sphere 2 at distance $r$ is given by

$$
\begin{aligned}
V\left(r, \phi_{01}, \phi_{02}\right)= & \frac{a^{4}}{4 \pi \epsilon_{0} \bar{\epsilon}} \int_{\pi / 2}^{\pi} \sin \left(\theta_{1}\right) d \theta_{1} \sin \left(\theta_{2}\right) d \theta_{2} \\
& \times \int_{0}^{2 \pi} d \phi_{1} d \phi_{2} \zeta\left(\theta_{1}, \phi_{2}\right) \zeta\left(\theta_{1}, \phi_{2}\right) \\
& \times\left[\frac{1}{\left|\mathbf{r}_{1}-\mathbf{r}_{2}+\mathbf{r}\right|}+\frac{1}{\left|\mathbf{r}_{1}^{\prime}-\mathbf{r}_{2}^{\prime}+\mathbf{r}\right|}\right. \\
& \left.-\frac{1}{\left|\mathbf{r}_{1}-\mathbf{r}_{2}^{\prime}+\mathbf{r}\right|}-\frac{1}{\left|\mathbf{r}_{1}^{\prime}-\mathbf{r}_{2}+\mathbf{r}\right|}\right],
\end{aligned}
$$

where $\mathbf{r}_{i}=\left(a, \theta_{i}, \phi_{i}\right)$ and $\mathbf{r}_{i}^{\prime}=\left(a+\lambda_{D}, \theta_{i}, \phi_{i}\right)$ are the radial vectors in the spherical coordinates and $\mathbf{r}=r \hat{\mathbf{x}}$ is the vector con- 
necting the two spheres. Because of thermal fluctuations, the orientation of the two dipoles with angles $\phi_{01}$ and $\phi_{02}$, respectively, can take arbitrary values. The effective interaction potential $U(r)$ between the two spheres after averaging over $\phi_{01}$ and $\phi_{02}$ at temperature $T$ is determined by [2]

$$
\frac{U(r)}{k_{B} T}=-\ln \left[\frac{1}{(2 \pi)^{2}} \int_{0}^{2 \pi} d \phi_{01} d \phi_{02} e^{-V\left(r, \phi_{01}, \phi_{02}\right) / k_{B} T}\right] .
$$

Finally, Eq. (10) is solved numerically.

Figure 13(a) shows the calculated $U(r) / k_{B} T$ as a function of the normalized particle separation $r / d$. In the calculation, we choose $\lambda_{D}=20 \mathrm{~nm}$ and $b=0.88$, which gives rise to a ratio of dipole moments $P_{\|} / P_{z} \simeq \pi b \sqrt{\epsilon} / 4 \simeq 6.2$. The value of the surface charge density $\zeta_{0}$ is varied from 1.1 to $0.78 \mu \mathrm{C} / \mathrm{cm}^{2}$, which are close to the experimental values. While the surface charge distribution used in the calculation is somewhat idealized, the resulting $U(r)$ proves instructive for our purpose. It has an energy barrier of height $\sim 6 k_{B} T$ at the particle separation $r_{1} \simeq 2.2 d$. For larger particle separations, $U(r)$ decays as $1 / r^{3}$. For smaller particle separations, the calculated $U(r)$ shows an attractive well, whose amplitude increases with $\zeta_{0}$. When $\zeta_{0}=1.1 \mu \mathrm{C} / \mathrm{cm}^{2}$, the attractive well has an amplitude of $-4 k_{B} T$ located at $r_{0} \simeq 1.3 d$. For even smaller separations $\left(r<r_{0}\right)$, the calculated $U(r)$ shows a steep repulsion, which is expected from the usual screened Coulomb potential as shown in Eq. (1). The characteristic features shown in Fig. 13(a) are all observed in the experiment with $r_{1} \sim \lambda_{N}$ given in Eq. (7).

Figure 13(b) shows how the interaction potential $U(r) / k_{B} T$ changes with the screening length $\lambda_{D}$. In the calculation, we choose $b=0.88$ and the value of $P_{z}$ is fixed, such that the calculated $U(r) / k_{B} T$ at large particle separations remains the same form $U(r) / k_{B} T \simeq 3200(d / r)^{3}$. Because $P_{z}$ depends on both $\zeta_{0}$ and $\lambda_{D}$, increasing $\lambda_{D}$ will result in a decrease in $\zeta_{0}$ for constant $P_{z}$. It is seen from Fig. 13(b) that as the value of $\lambda_{D}$ increases, the location $r_{0}$ of the attractive well shifts toward a larger value of $r$.

To examine how the vertical position of the particles at the interface affects the interaction potential, we calculate $U(r)$ for charged spheres with $2 / 3$ diameter immersed in water. As will be shown below, this particle position is closer to the actual experimental situation. Using the same surface charge density $\zeta(\theta, \phi)$ given in Eq. (8) with $\sin ^{-1}(1 / 3) \leqslant \theta$ $\leqslant \pi$ and $0 \leqslant \phi \leqslant 2 \pi$, we find the out-of-plane dipole moment $P_{z} \simeq 0.89 \pi a^{2} \zeta_{0} \lambda_{D} / \sqrt{\epsilon}$ and the in-plane dipole moment $P_{\|}$ $\simeq(3 \pi / 10) b \pi a^{2} \zeta_{0} \lambda_{D}$. As the interfacial particle is pulled further into the aqueous phase, $P_{z}$ decreases whereas $P_{\|}$increases and thus the ratio of the two dipole moments becomes $P_{\|} / P_{z} \simeq 0.34 \pi b \sqrt{\epsilon}$. Figure 14 shows the calculated $U(r) / k_{B} T$ versus $r / d$ at fixed $b=0.64$, from which we have $P_{\|} / P_{z} \simeq 6$.1. Similar to Fig. 13(a), the calculated $U(r)$ at $r$ $\simeq r_{0}$ shows an attractive well, whose amplitude increases with $\zeta_{0}$ (triangles versus diamonds). When the value of $\lambda_{D}$ is increased from 2 to $50 \mathrm{~nm}$ (triangles versus circles), the location $r_{0}$ of the attractive well shifts toward a larger value of $r$.

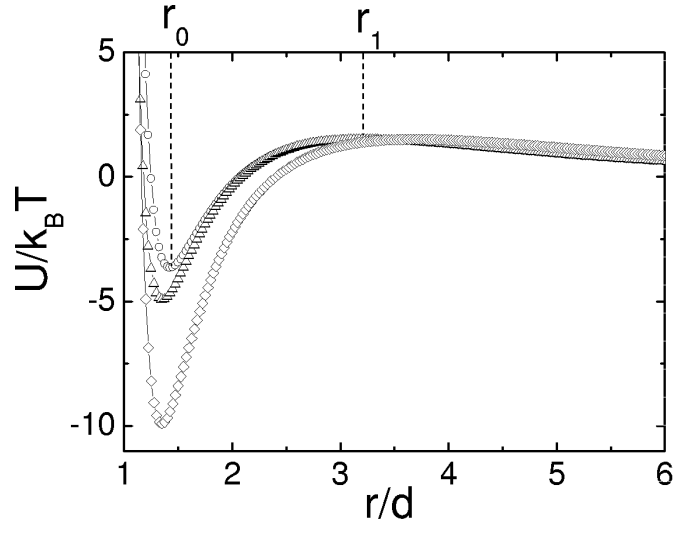

FIG. 14. Calculated interaction potential $U(r) / k_{B} T$ as a function of the normalized particle separation $r / d$ for charged spheres with $2 / 3$ diameter immersed in water. The calculation is done with fixed $b=0.65$ but varying Debye-Huckel screening length and surface charge density $\lambda_{D}=50 \mathrm{~nm}, \zeta_{0}=0.22 \mu \mathrm{C} / \mathrm{cm}^{2}$ (circles), $\lambda_{D}=2 \mathrm{~nm}$, $\zeta_{0}=5.4 \mu \mathrm{C} / \mathrm{cm}^{2}$ (triangles), and $\lambda_{D}=2 \mathrm{~nm}, \quad \zeta_{0}=6.6 \mu \mathrm{C} / \mathrm{cm}^{2}$ (diamonds).

\section{Electrostatic energy of bonded particle clusters}

A theoretical challenge is whether the dipole interaction can explain the interesting structure of various bonded particle clusters shown in Figs. 7 and 9. We note that this is not an easy task, not only because the PS spheres are not all identical in the surface charge distribution, but also because the stability of the structures depends not only on the longranged dipole interaction, but also on the existence of a short-ranged repulsion between these spheres. To see this, let us assume that for a particle cluster at configuration $\left\{\vec{r}_{i}, \vec{p}_{i}\right\}$, the energy of the system,

$$
E\left[\left\{\vec{r}_{i}, \vec{p}_{i}\right\}\right]=\sum_{i, j} \frac{1}{r_{i j}^{3}}\left[\vec{p}_{i} \cdot \vec{p}_{j}-3\left(\vec{p}_{i} \cdot \hat{n}_{i j}\right)\left(\vec{p}_{j} \cdot \hat{n}_{i j}\right)\right]
$$

is negative, where $r_{i j}=\left|\vec{r}_{i}-\vec{r}_{j}\right|, \hat{n}_{i j}=\left(\vec{r}_{i}-\vec{r}_{j}\right) / r_{i j}$, and $\vec{p}_{i}$ is the dipole moment at position $\vec{r}_{i}$. This energy can be further reduced by simply scaling $\vec{r}_{i} \rightarrow \gamma \vec{r}_{i}$, for $\forall i$, with $\gamma<1$. The system can only be stabilized if there is a short-ranged repulsion between the latex spheres, which prevents the system from further shrinking. The screened Coulomb interaction shown in Eq. (6) provides this necessary repulsion for the bonded particle clusters. The existence of both long-ranged and short-ranged interactions between the PS spheres makes the energetic analysis difficult except for some simple configurations.

A simple configuration, which is locally stable (a local minimum in the energy landscape $E\left[\left\{\vec{r}_{i}, \vec{p}_{i}\right\}\right]$ ), is when all the spheres are aligned in a straight line $\vec{L}$ with all dipoles pointing along $\vec{L}$. The distance between adjacent spheres is determined by the short-ranged repulsion, which we assume to be hard-sphere-like. Assuming that all the PS spheres are identical, we find that for small perturbations the energy of a particle chain is of the form 


$$
\begin{aligned}
E\left[\left\{\vec{r}_{i}, \vec{p}_{i}\right\}\right]= & E_{0}+\sum_{i, j} \frac{p^{2}}{r_{i j}^{3}}\left[\frac{6}{r_{i j}^{2}}\left(\delta \vec{r}_{i}-\delta \vec{r}_{j}\right)^{2}+\frac{1}{2}\left[\left(\delta \theta_{i}+\delta \theta_{j}\right)^{2}\right.\right. \\
& \left.\left.+3\left(\delta \theta_{i}^{2}+\delta \theta_{j}^{2}\right)\right]\right]
\end{aligned}
$$

where $E_{0}=-2 N p^{2} \zeta(3) /(2 a)^{3}$ is the energy of the straight line configuration of $N$ spheres. Here $p$ is the dipole moment, $\zeta(n)=\sum_{k=1}^{\infty} k^{-n}$ is the Riemann Zeta function, $2 a$ is the centerto-center separation between adjacent spheres, $\delta \vec{r}_{i}$ is a small change in position of the $i$ th dipole, and $\delta \theta_{i}$ is the corresponding change in dipole orientation. In the above, we have assumed that the dipoles are all lying on the plane of the interface and $\theta_{i}$ is the angle between the $i$ th dipole and $\vec{L}$. Notice that for close packing, $\delta \vec{r}_{i}$ 's must be perpendicular to $\vec{L}$. Equation (11) clearly shows that the straight line configuration is a local minimum in energy.

Other chainlike configurations can be studied with Eq. (11) if $\delta \vec{r}_{i}$ 's are slowly changing functions of $\vec{r}_{i}$. For simplicity, we assume $\delta \theta_{i} \simeq 0$ (i.e., the dipoles are always aligned along the line of spheres) and $\delta \vec{r}_{i} \simeq \delta \vec{r}_{j}+\vec{r}_{i j} \cdot \partial \delta \vec{r}_{j} / \partial \vec{r}_{j}$. Putting these back into Eq. (11), we obtain

$$
E\left[\left\{\vec{r}_{i}, \vec{P}_{i}\right\}\right] \rightarrow E_{0}+\alpha \sum_{i}\left(\frac{\partial \delta \vec{r}_{i}}{\partial \vec{r}_{i}}\right)^{2},
$$

where $\alpha=6 p^{2} \sum_{j} r_{i j}^{-3}=6 p^{2} \zeta(3) /(2 a)^{3}$. The above energy can be minimized for various chain configurations with a fixed end-to-end distance. In this case, $\partial \delta \vec{r}_{i} / \partial \vec{r}_{i}=$ const gives a minimum energy state corresponding to a chain with constant curvature. This situation is in analogy with free particles in Newtonian mechanics, where $L=\int_{\vec{x}_{1}}^{\vec{x}_{2}} d t(d \vec{x} / d t)^{2}$ and $d \vec{x} / d t=$ const corresponding to a string with constant curvature. In particular, when the end-to-end distance is zero (closed chains), circular chains are the lowest-energy configurations. As discussed in Sec. III, circular chains are commonly found in the experiment and our analysis suggests that these structures indeed result from a dipolar attraction.

Another piece of evidence that the interaction between the PS spheres is dipolar in nature is that structures with one or more spheres stuck to the outside of the circular chains are seldom observed. (Notice that spheres can be trapped inside a circular chain without attraction.) For isotropic attractive interactions, a straight line of spheres can also minimize energy locally. However, extra spheres can "stick" to the line of spheres easily in this case. This is not the case for dipolar interactions. The energy gain by sticking an extra sphere to a line of spheres is a small fraction of the energy $-6 p^{2} \zeta(3) /(2 a)^{3}$ because of frustration in dipole directions. In our experiment, we have $6 p^{2} \zeta(3) /(2 a)^{3} \sim k_{B} T$, and thus the probability for an extra sphere to stick to a line of spheres is very small.

\section{Comparison with other models}

A unique feature that distinguishes dipole attraction from other interactions, such as capillary forces and attractions resulting from wetting and surface roughness of the particles $[13,18,21,28-32]$, is that the dipole attraction is anisotropic in the plane of the interface. The formation of chainlike structures shown in Figs. 7 and 9, therefore, effectively refutes those previously proposed mechanisms, predicting isotropic interaction potentials in the plane of the interface. There is a class of theoretical models that predicts the existence of an energy well but no energy barrier. For example, the usual capillary effects cannot give an energy barrier at a separation of a few particle diameters, because they occur only near the contact line between the particle surface and the water-air interface.

In the following, we clarify several issues that often arise in the discussion of the interfacial particles. First, the weight of the PS spheres used in the experiment does not cause deformations of the interface. Pieranski [23] showed that the surface energy for an interfacial particle has the form

$$
F_{s}=\pi a^{2} \sigma_{w / a}\left[\tilde{z}^{2}+2(\alpha-\beta) \tilde{z}+2 \alpha+2 \beta-1\right],
$$

where $\tilde{z}=z / a$ is the vertical position of the center of the particle with respect to the interface normalized by the particle radius $a$, and $\alpha=\sigma_{p / a} / \sigma_{w / a} \simeq 0.49$ and $\beta=\sigma_{p / w} / \sigma_{w / a}$ $\simeq 0.14$ are, respectively, the surface tensions of the polystyrene/air interface and the polystyrene/water interface [48], both normalized by the surface tension of the water/air interface. By staying at the interface, the PS spheres gain surface energy, because $\sigma_{w / a}$ is larger than $\sigma_{p / a}$ and $\sigma_{p / w}$. Minimizing the surface energy with respect to $\widetilde{z}$, one finds the equilibrium position of the PS spheres $\widetilde{z}_{0}=\beta-\alpha=-0.35$. This calculation suggests that $\sim 2 / 3$ of the particle (by diameter) is immersed in water and the remaining $1 / 3$ is in air. Because the characteristic energy scales as $\pi a^{2} \sigma_{w / a}$, which is $10^{7}$ times larger than $k_{B} T$, the PS spheres are strongly bound to the interface. While the Koehler illumination used in the inverted microscope does not allow us to directly determine the vertical position of the interfacial particles, we find that these particles remain at sharp focus under high magnification, indicating that the vertical position of the PS spheres is indeed determined by a sharp surface energy minimum much larger than $k_{B} T$.

The surface energy $\pi a^{2} \sigma_{w / a}$ is also $10^{7}$ times larger than the gravitational energy $\Delta m g a$ of a PS sphere of radius $a$ and buoyant mass $\Delta m$, where $g$ is the gravitational acceleration. As a result, the interfacial particles can move freely in the vertical direction and choose their equilibrium position $\widetilde{z}_{0}$, at which the contact angle between the spherical particle surface and the flat interface satisfies Young's equation simultaneously. No additional wetting of the particles is needed to balance the surface forces. In other words, bending the interface takes much more energy than moving the interfacial particles vertically.

Second, surface roughness effects are not likely to cause macroscopic deformations of the interface. Figures 10 and 11 clearly show that the surface of the PS spheres used in the experiment is smooth down to a few nanometers. Therefore, deformations of the interface due to surface roughness pinning cannot happen on the micrometer or submicrometer scales. If nanoscale pinning occurs, the nanoscopic pinning sites will be averaged out spatially over an $\sim 1000$-nm-long circular contact line between the spherical particle surface and the flat interface. As a result, the nanoscopic pinning is 
simply too small to affect a micrometer-sized particle at the interface. The chemical heterogeneities on the particle surface, which are revealed by the AFM phase images shown in Figs. 10 and 11, however, might cause interface pinning or meniscus deformation. Whether this effect can produce a large enough dipolelike attraction as discussed above requires further theoretical investigation. Finally, Megens and Aizenberg [33] showed that the electrocapillary forces proposed by Nikolaides et al. [21], which may cause interface deformation, are too small for the observed attraction between the interfacial particles.

\section{CONCLUSION}

We have carried out a systematic study of interactions between charged polystyrene latex spheres at the water-air interface. Two kinds of polystyrene (PS) spheres are used in the experiment. One has anionic (negative) carboxyl groups on the surface with diameter $d=1.1 \mu \mathrm{m}$ and (nominal) surface charge density $\zeta_{0}=12.5 \mu \mathrm{C} / \mathrm{cm}^{2}$. The second kind of PS spheres has anionic sulfate groups on the surface with diameter $d=1.0 \mu \mathrm{m}$ and $\zeta_{0}=2.8 \mu \mathrm{C} / \mathrm{cm}^{2}$. An important objective of the paper is to delineate the experimental conditions and procedures, including cleaning of the interface and purification of the colloidal samples, under which one can obtain accurate and reliable data for the measurement of the interaction potential between the interfacial particles. Because the interactions and dynamics of the interfacial particles are extremely sensitive to impurities at the interface, the experimental conditions and procedures described in the paper are essential to produce a well-dispersed monolayer of particles and carry out well-controlled measurements at the interface.

Direct optical observations reveal how the PS spheres attract and form "Coulombic molecules" at the interface. As in the formation of ordinary molecules, the symmetry of the bonded particle clusters (equivalent of molecular symmetry groups), the large interparticle separations (equivalent of molecular bond length), and the unique experimental conditions under which one obtains two distinct equilibrium particle configurations provide robust and compelling evidence for the dipolelike attraction at the interface. From the optical measurements, we arrive at the following qualitative picture for the interaction potential $U(r)$ between the interfacial particles. As depicted in Fig. 13(a), it has an energy barrier of height $5-10 k_{B} T$ at $r_{1}$ and decays as $1 / r^{3}$ for larger $r\left(>r_{1}\right)$. For smaller particle separations, $U(r)$ has a deep energy well of the order of $10 k_{B} T$ at $r_{0}$. For even smaller separations $(r$ $<r_{0}$ ), the usual screened Coulomb repulsion becomes dominant.

Atomic force microscopy (AFM) is used to examine the actual distribution of charge groups on the colloidal surface. AFM phase images obtained under tapping mode show patchy domains on the surface of the PS spheres, indicating that their surface charge distribution is not uniform as is commonly believed. Such patchy charges can introduce fluctuating in-plane dipoles, leading to an attraction at short interparticle distances and formation of stable bonded particle clusters at the interface. The surface heterogeneity is manifested most effectively at the interface, at which the particle interaction becomes very sensitive to the asymmetry of the surface charge distribution.

The nonuniform surface charge distribution of the PS spheres is modeled by a simple dipolar distribution $\zeta(\theta, \phi)$ $=\zeta_{0}\left[1+b \cos \left(\phi-\phi_{0}\right)\right]$. With this hypothetical nonuniform surface charge distribution, we calculate the electrostatic potential between two identical spheres numerically and obtain the effective interaction potential $U(r)$. While the surface charge distribution used in the calculation is somewhat idealized, the resulting $U(r)$ proves instructive for our purpose. The characteristic features of the calculated $U(r)$ agree well with the experimental results. In particular, the calculated $U(r)$ at small particle separations shows an attractive well, whose amplitude increases with the average surface charge density $\zeta_{0}$. A further theoretical analysis on the electrostatic energy of the bonded particle clusters suggests that circular chainlike particle clusters that are commonly observed in the experiment indeed result from a minimization of dipolar attractions. The dipole-attraction mechanism resulting from a nonuniform surface charge distribution casts new light on the understanding of attractions between like-charged species occurring in other colloidal systems. Certainly, a more detailed modeling of $\zeta(\theta, \phi)$ is needed in order to calculate $U(r)$ for realistic polystyrene latex spheres.

\section{ACKNOWLEDGMENTS}

We are grateful for useful discussions and communications with D. Weitz, D. Grier, P. Chaikin, and J. Goodwin. We thank O. K. C. Tsui for letting us use her LangmuirBlodgett trough. This work was supported by the Research Grants Council of Hong Kong SAR under Grants No. HKUST603305 (P.T.) and No. HKUST602803 (T-K.N.) and by the U.S. National Science Foundation under Grant No. EPS-0132534 (W.T.F.).
[1] W. B. Russel, D. A. Saville, and W. R. Schowalter, Colloidal Dispersion (Cambridge University Press, Cambridge, UK, 1989).

[2] J. Israelachvili, Intermolecular and Surface Forces, 2nd ed. (Academic Press, San Diego, 1991).

[3] P. Attard, Curr. Opin. Colloid Interface Sci. 6, 366 (2001).

[4] D. G. Grier and Y. Han, J. Phys.: Condens. Matter 16, S4145
(2004).

[5] N. Ise, T. Okubo, M. Sugimura, K. Ito, and H. J. Nolte, J. Chem. Phys. 78, 536 (1983).

[6] G. M. Kepler and S. Fraden, Phys. Rev. Lett. 73, 356 (1994).

[7] M. D. Carbajal-Tinoco, F. Castro-Roman, and J. L. ArauzLara, Phys. Rev. E 53, 3745 (1996).

[8] A. E. Larsen and D. G. Grier, Nature (London) 385, 230 
(1997).

[9] Y. Han and D. G. Grier, Phys. Rev. Lett. 91, 038302 (2003).

[10] T. E. Angelini, H.-J. Liang, W. Wriggers, and G. C. Wong, Proc. Natl. Acad. Sci. U.S.A. 100, 8634 (2003).

[11] W. M. Gelbart, R. F. Bruinsma, P. A. Pincus, and V. A. Parsegian, Phys. Today 53(9), 38 (2000).

[12] I. Sogami and N. Ise, J. Chem. Phys. 81, 6320 (1984).

[13] X. Chu and D. T. Wasan, J. Colloid Interface Sci. 184, 268 (1996).

[14] J. C. Neu, Phys. Rev. Lett. 82, 1072 (1999).

[15] J. E. Sader and D. Y. C. Chan, J. Colloid Interface Sci. 213, 268 (1999); Langmuir 16, 324 (2000).

[16] E. Trizac, Phys. Rev. E 62, R1465 (2000); E. Trizac and J.-L. Raimbault, ibid. 60, 6530 (1999).

[17] T. M. Squires and M. P. Brenner, Phys. Rev. Lett. 85, 4976 (2000).

[18] B. I. Ivlev, J. Phys.: Condens. Matter 14, 4829 (2002).

[19] F. Ghezzi and J. C. Earnshaw, J. Phys.: Condens. Matter 9, L517 (1997).

[20] J. Ruiz-Garcia, R. Gamez-Corrales, and B. I. Ivlev, Phys. Rev. E 58, 660 (1998).

[21] M. G. Nikolaides, A. R. Bausch, M. F. Hsu, A. D. Dinsmore, M. P. Brenner, C. Gay, and D. A. Weitz, Nature (London) 420, 299 (2002).

[22] O. Gomex-Guzman and J. Ruiz-Garcia, J. Colloid Interface Sci. 291, 1 (2005).

[23] P. Pieranski, Phys. Rev. Lett. 45, 569 (1980).

[24] A. J. Hurd, J. Phys. A 18, L1055 (1985).

[25] G. Y. Onoda, Phys. Rev. Lett. 55, 226 (1985).

[26] N. D. Denkov, O. D. Velev, P. A. Kralchevsky, and I. B. Ivanov, Nature (London) 361, 26 (1993).

[27] H. H. Wickmann and J. N. Korley, Nature (London) 393, 445 (1998).

[28] D. Y. C. Chan, J. D. Henry, and L. R. White, J. Colloid Interface Sci. 79, 410 (1981).

[29] P. A. Kralchevsky, V. N. Paunov, I. B. Ivanov, and K. Nagayama, J. Colloid Interface Sci. 151, 79 (1992).
[30] D. C. Morse and T. A. Witten, Europhys. Lett. 22, 549 (1993).

[31] P. A. Kralchevsky and N. D. Denkov, Curr. Opin. Colloid Interface Sci. 6, 383 (2001).

[32] D. Stamou, C. Duschl, and D. Johannsmann, Phys. Rev. E 62, 5263 (2000).

[33] M. Megens and J. Aizenberg, Nature (London) 424, 1014 (2003).

[34] J. C. Fernandez-Toledano, A. Moncho-Jorda, F. MartinezLopez, and R. Hidalgo-Alvarez, Langmuir 20, 6977 (2004).

[35] W. Chen, S. Tan, T.-K. Ng, W. T. Ford, and P. Tong, Phys. Rev. Lett. 95, 218301 (2005).

[36] Langmuir-Blodgett Troughs Operating Manual, 6th ed. (Nima Technology Ltd., UK, 2001).

[37] S. H. Behrens and D. G. Grier, Phys. Rev. E 64, 050401(R) (2001).

[38] M. Brunner, C. Bechinger, W. Strepp, V. Lobaskin, and H. H. von Grunberg, Europhys. Lett. 58, 926 (2002).

[39] R. Aveyard, B. P. Binks, J. H. Clint, P. D. I. Fletcher, T. S. Horozov, B. Neumann, V. N. Paunov, J. Annesley, S. W. Botchway, D. Nees, A. W. Parker, A. D. Ward, and A. N. Burgess, Phys. Rev. Lett. 88, 246102 (2002).

[40] A. San Paulo and R. Garcia, Phys. Rev. B 64, 193411 (2001).

[41] R. W. Stark, G. Schitter, and A. Stemmer, Phys. Rev. B 68, 085401 (2003).

[42] S. Tan, R. L. Sherman, D. Qin, and W. T. Ford, Langmuir 21, 43 (2005).

[43] S. Alexsander, P. M. Chaikin, P. Grant, G. J. Morales, and P. Pincus, J. Chem. Phys. 80, 5776 (1984).

[44] S. H. Behrens and D. G. Grier, J. Chem. Phys. 115, 6716 (2001).

[45] E. Trizac, L. Bocquet, and M. Aubouy, Phys. Rev. Lett. 89, 248301 (2002).

[46] M. Aubouy, E. Trizac, and L. Bocquet, J. Phys. A 36, 5835 (2003).

[47] Y. Zhou et al. (unpublished).

[48] E. Sheppard and N. Tcheurekdjian, J. Colloid Interface Sci. 28, 181 (1968). 